\title{
Magnetization of carbonaceous asteroids by nebular fields and the origin of CM chondrites
}

\section{Samuel Courville ( $\square$ swcourvi@asu.edu )}

Arizona State University https://orcid.org/0000-0002-9539-1301

Joseph O'Rourke

Arizona State University https://orcid.org/0000-0002-1180-996X

Julie Castillo-Rogez

Jet Propulsion Laboratory, California Institute of Technology

\section{Roger Fu}

Harvard University

\section{Rona Oran}

Massachusetts Institute of Technology

\section{Benjamin Weiss}

MIT https://orcid.org/0000-0003-3113-3415

\section{Linda Elkins-Tanton}

Arizona State University

\section{Article}

Keywords:

Posted Date: January 12th, 2022

DOI: https://doi.org/10.21203/rs.3.rs-1196868/v1

License: (c) (1) This work is licensed under a Creative Commons Attribution 4.0 International License.

Read Full License

Version of Record: A version of this preprint was published at Nature Astronomy on October 13th, 2022. See the published version at https://doi.org/10.1038/s41550-022-01802-z. 


\section{Magnetization of carbonaceous asteroids by nebular 2 fields and the origin of $\mathrm{CM}$ chondrites}

3

4 Samuel W. Courville ${ }^{1 *}$, Joseph G. O'Rourke ${ }^{1}$, Julie C. Castillo-Rogez ${ }^{2}$, Roger R. Fu ${ }^{3}$, Rona

5 Oran $^{4}$, Benjamin P. Weiss ${ }^{4}$, Linda T. Elkins-Tanton ${ }^{1}$

6

8

8
9

9
10

11

12
${ }^{1}$ School of Earth and Space Exploration, Arizona State University, Tempe, AZ, USA,

${ }^{2}$ Jet Propulsion Laboratory, California Institute of Technology, Pasadena, CA, USA, ${ }^{3}$ Department of Earth and Planetary Sciences, Harvard University, Cambridge, MA, USA, ${ }^{4}$ Department of Earth, Atmospheric and Planetary Sciences, MIT, Cambridge, MA, USA

*swcourvi@asu.edu 


\section{Introductory Paragraph:}

14 The solar nebula carried a strong magnetic field that had a stable intensity and direction for

15 periods of a thousand years or more ${ }^{1}$. The solar nebular field may have produced post-accretional

16 magnetization in at least two groups of meteorites, $\mathrm{CM}$ and $\mathrm{CV}$ chondrites ${ }^{1-3}$, which originated

17 from planetesimals that may have underwent aqueous alteration before gas in the solar nebula

18 dissipated ${ }^{1,3}$. Magnetic minerals produced during aqueous alteration, such as magnetite and

19 pyrrhotite $^{4}$, could acquire a chemical remanent magnetization from that nebular field ${ }^{3}$. However,

20 many questions about the size, composition, formation time, and, ultimately, identity of the

21 parent bodies that produced magnetized $\mathrm{CM}$ and $\mathrm{CV}$ chondrites await answers-including

22 whether a parent body might exhibit a detectable magnetic field today. Here, we use thermal

23 evolution models to show that planetesimals that formed between a few Myr after CAIs and $\sim 1$

24 Myr before the nebular gas dissipated could acquire from the nebular field, and retain until

25 today, a chemical remanent magnetization throughout nearly their entire volume. Hence, in-situ

26 magnetometer measurements of C-type asteroids could help link magnetized asteroids to

27 magnetized meteorites. Specifically, a future mission could search for a magnetic field as part of

28 testing the hypothesis that 2 Pallas is the parent body of the CM chondrites 5 . Overall, large

29 carbonaceous asteroids might record ancient magnetic fields in magnetic remanence that

30 produces strong modern magnetic fields, even without a metallic core that once hosted a

31 dynamo. 


\section{Main:}

Several meteorites in two groups of carbonaceous chondrites, the CM and CV chondrites,

35 host a chemical remanent magnetization $(\mathrm{CRM})^{3}$ and possibly also thermoremanent

36 magnetization ${ }^{6}$. CRM in a meteorite implies that chemical reactions produced ferromagnetic

37 minerals within the meteorite's parent body while in the presence of a strong and temporally

38 stable magnetic field. CM and CV chondrites experienced aqueous alteration, but little or no

39 further dehydration or melting ${ }^{4}$. Aqueous alteration can produce minerals that are possible

40 magnetic carriers ${ }^{4}$, such as magnetite $\left(\mathrm{Fe}_{3} \mathrm{O}_{4}\right)$ and pyrrhotite $\left(\mathrm{Fe}_{(1-\mathrm{x})} \mathrm{S}, \mathrm{x}=0\right.$ to 0.2$)$. In general,

41 ferromagnetic minerals forming in the presence of a background magnetic field can acquire a

$42 \mathrm{CRM}$ in the same direction as the background field ${ }^{7,8}$. Thus, CRM in CM and CV chondrites

43 indicates that their parent bodies may have undergone aqueous alteration in the presence of a

44 strong magnetic field.

45

The magnetic field sustained by the cloud of dust and gas in the early solar nebula is

47 likely the source of magnetization for the parent bodies of the CM chondrites ${ }^{3}$. Based on

48 telescopic observations of distant solar nebulae and thermal remanent magnetization within

49 chondrules of primitive LL and CO chondrites, the early solar nebula likely had a strong

50 magnetic field that was active in the first few Myr of the solar system ${ }^{2,9-11}$. The nebula likely

51 supported a strong magnetic field until at most 3.9 Myr after CAIs in the inner solar system, or

52 at most $\sim 4.8 \mathrm{Myr}$ in the outer solar system ${ }^{1}$. Thermal evolution models indicate whether

53 planetesimals could experience aqueous alteration before the nebula dissipated - and thus

54 whether planetesimals could acquire and retain magnetization from the nebular field. 
During the heating of a planetesimal's interior through the radioactive decay of ${ }^{26} \mathrm{Al}$, two

57 important thermal events determine the potential for acquiring a chemical remanent

58 magnetization. First, the planetesimal reaches the melting point of water ice $(\sim 273 \mathrm{~K})$. When

59 water ice melts, liquid water drives aqueous alteration and produces the magnetic carriers

60 magnetite and pyrrhotite ${ }^{4}$. If formed in the presence of the nebular magnetic field, these minerals

61 acquire CRM. The second event occurs if the planetesimal reaches a maximum unblocking

62 temperature, above which magnetized domains would randomize in zero-field conditions in a

63 time less than the duration of peak heating (as short as $\sim 10^{2} \mathrm{kyr}$ ). This maximum unblocking

64 temperature, henceforth called the unblocking temperature, is different for each mineral, and also

65 depends on geometric properties of individual grains such as size and shape ${ }^{12}$. Since these

66 properties are stochastic and unknown a priori, we adopt fixed unblocking temperatures that are

67 within empirically measured ranges ${ }^{3}: 550 \mathrm{~K}$ for pyrrhotite and $850 \mathrm{~K}$ for magnetite (vs. Curie

68 temperatures of $583 \mathrm{~K}$ and $858 \mathrm{~K}$, respectively). If the planetesimal heats above the unblocking

69 temperature(s) of its magnetic carrier(s) after the nebular magnetic field dissipates and stays

70 above that temperature for longer than $\sim 10^{2} \mathrm{kyr}$, then any magnetization in the minerals would

71 be reset as the minerals re-magnetize in zero-background-magnetic-field conditions. If the

72 planetesimal heats above, but then cools below the unblocking temperature(s) prior to the end of

73 the lifetime of the nebula, then it will acquire a thermoremanence, possibly in addition to CRM.

74

75 An undisturbed planetesimal may experience one of three thermal evolution cases

76 depending on the timing of the aforementioned milestones with respect to the lifetime of the

77 solar nebular magnetic field. First, a planetesimal may form too late to reach the temperature to

78 melt water ice before the solar nebula dissipates (Fig. 1a). This case results in no nebular 
79 magnetization. Second, a planetesimal may form early enough to undergo aqueous alteration

80 before the nebula dissipates but does not subsequently heat beyond the unblocking temperatures

81 of magnetite or pyrrhotite (Fig. 1b). This scenario could lead to magnetization of nearly the

82 entire planetesimal. Lastly, a planetesimal could form early enough to undergo aqueous

83 alteration, but subsequently heat beyond the unblocking temperatures (Fig. 1c). Our models

84 indicate that an undisturbed planetesimal (i.e., that did not experience impact unroofing and rapid

85 cooling $^{13}$ ) that heats beyond the unblocking temperature does not cool below that threshold again

86 until many Myrs past when the nebula dissipates. Thus, such a planetesimal that reaches the

87 unblocking temperature before the nebula dissipates would not retain magnetization throughout

88 its interior. However, a thin shell of aqueously altered, chondritic material that does not reach the

89 unblocking temperature could retain magnetization near the surface. Thus, magnetized CM

90 chondrite meteorites could originate from either the second or third case. Only the second case

91 could result in an asteroid with large-scale remanent magnetization from the solar nebula.

Figure 2 plots the maximum volume percentage of a planetesimal that could be

94 magnetized as a function of the planetesimal's radius and formation time. If the primary

95 magnetic carrier is pyrrhotite, we find that a planetesimal may acquire large-scale magnetization

96 from the solar nebula if it formed between $\sim 3.5$ and 3.7 Myr after CAIs, depending on the exact

97 timing of nebular dissipation (Fig. 2a). Since magnetite has a higher unblocking temperature,

98 earlier forming planetesimals that reach hotter temperatures could still retain magnetization. If

99 magnetite is abundant as a magnetic carrier, then we find that large-scale magnetization is

100 possible for formation times between $\sim 2.7$ and 3.7 Myr after CAIs (Fig. 2b). These ranges are

101 consistent with the formation time expected for the parent bodies of the $\mathrm{CM}$ chondrites ${ }^{14}$. 
102 Assuming that the entire volume of possible magnetization in each planetesimal model is

103 coherently magnetized at the strength observed in the CM chondrites (see below for caveats),

$104 \sim 10^{-4} \mathrm{~A} \mathrm{~m}^{2} / \mathrm{kg}$, we plot the maximum possible magnetic dipole moment that each planetesimal

105 might have today (Figs. 2c and 2d). For example, if Pallas formed at $\sim 3.5 \mathrm{Myr}$ after CAIs, its

106 maximum possible dipole moment now is $\sim 10^{16} \mathrm{~A} \mathrm{~m}^{2}$. For comparison, Earth's magnetic

107 moment is $\sim 8 \times 10^{22} \mathrm{~A} \mathrm{~m}^{2}$ and the metallic asteroid 16 Psyche may have a moment between

$108 \sim 10^{15} \mathrm{~A} \mathrm{~m}^{2}$ and $10^{17} \mathrm{~A} \mathrm{~m}^{2}$ if it is a disrupted planetary core ${ }^{15}$.

The maximum possible dipole moments displayed in Figs. $2 \mathrm{c}$ and $2 \mathrm{~d}$ assume an idealistic

111 scenario wherein the entire volume of the planetesimal has been magnetized unidirectionally.

112 However, aqueous alteration need not occur homogeneously and contemporaneously throughout

113 a planetesimal. If the solar nebular field's intensity and direction change between the time at

114 which two different regions of a planetesimal experience aqueous alteration, then these two

115 different regions would acquire different magnetization directions. The number and size of

116 regions with differing magnetization strengths would depend on the rate of alteration compared

117 to rate of field change. Additionally, impacts could scramble regions of coherent magnetization.

118 The effective dipole moment of the planetesimal would be a function of the length scale of its

119 magnetized regions. The smaller the length scale, the lower the effective dipole moment.

The asteroid 2 Pallas is a candidate parent body for the $\mathrm{CM}$ chondrites ${ }^{5}$ because it

122 exhibits a hydrated spectral signature in the $3-\mu \mathrm{m}$ region, which matches $\mathrm{CM}$ chondrite spectra ${ }^{16}$.

123 Impacts can produce the observed differences between the visible and near-infrared spectra of

124 Pallas and the CM chondrites ${ }^{5}$. Furthermore, the bulk density of Pallas $\left(\sim 2.9 \mathrm{~g} / \mathrm{cm}^{3}\right)$ is similar to 
125 the grain density of $\mathrm{CM}$ chondrites ${ }^{17}$. Pallas also appears to lack a thick icy shell, based on its

126 global morphology ${ }^{5}$, unlike Ceres. A possible model for Pallas' evolution is that it underwent

127 aqueous alteration on a global scale and subsequently lost the remaining water ice as a

128 consequence of subcatastrophic impacts, as suggested by the large basins found on its surface ${ }^{5,18}$.

129 An early phase of hydrothermal evolution could limit heating and separation of a metallic core ${ }^{5}$.

130 Based on our thermal evolution models, if Pallas is the parent body of the CM chondrites, then

131 we expect it to be measurably magnetized.

If the solar nebular field magnetized Pallas, then a spacecraft could possibly detect large-

134 scale remanent fields that have persisted until today. To assess detectability, we estimate the 135 magnetopause distance of a spherical asteroid with a radius of $256 \mathrm{~km}$ - the effective volume 136 radius of Pallas ${ }^{5,19}$ - orbiting at a distance of $2.8 \mathrm{AU}$ in the present solar wind. Fig. 3 calculates

137 the magnetopause distance as a function of the asteroid's effective magnetic dipole moment. This 138 calculation generally agrees with magnetopauses numerically observed in hybrid simulations of 139 asteroid magnetospheres in the solar wind ${ }^{20,21}$. For effective dipole moments above $\sim 10^{13}$ to $10^{14}$ $140 \mathrm{~A} \mathrm{~m}^{2}$, a spacecraft magnetometer flyby or orbiter could detect the asteroid's magnetosphere.

141 Orbiters or landers could detect magnetization at smaller length scales. The exact minimum

142 moment that is detectable depends on the specific mission design, and the orientation and 143 interaction of the magnetized regions with the solar wind. Furthermore, low magnetic moments 144 could also possibly be detected from orbit via the observation of Alfven waves ${ }^{22}$. The effective magnetic moment depends on the length scale of magnetization (Fig. 4).

147 The length scale of a planetesimal's magnetic field depends on how aqueous alteration occurred 
148 and whether the body has been disrupted by impacts throughout its lifetime. Different models of

149 aqueous alteration predict various upper bounds for the length scales of magnetization (Fig. 4). If

150 the distance over which fluid flows during the time that the solar nebular field is coherent

151 determines the length scale, then the effective permeability of the planetesimal is the critical

152 parameter. Assuming exhalation alteration (i.e., single-pass fluid flow directed to the surface ${ }^{23}$ )

153 and effective permeabilities between $\sim 10^{-13}$ and $10^{-12}$, we expect magnetization length scales of

$154 \sim 10-100 \mathrm{~km}$. Alternatively, if aqueous alteration occurs over multiple-pass, pore-fluid

155 convection, then the length and time scale of convection could determine the magnetization

156 length scale. Pore-fluid and/or hydrothermal convection could have length scales of $\sim 10 \mathrm{~km}$ but

157 requires a steep thermal gradient, which would only exist near the surface ${ }^{24,25}$. If alteration

158 occurs as the result of effusive events of high temperature fluid and steam escaping the

159 subsurface, i.e., a fumarolic system, then alteration would likely occur in distinct separate events

160 and have scales of less than $10 \mathrm{~km}$, perhaps consistent with alteration environments in terrestrial

161 ocean spreading centers ${ }^{26}$. For whole-body "mudball" convection ${ }^{27}$, the length scale could be as

162 great as $100 \mathrm{~km}$ if alteration occurs homogeneously throughout convective cells within the

163 mudball. Alternatively, aqueous alteration and the production of magnetic minerals may not be

164 dependent on fluid flow, i.e., in models with isochemical alteration ${ }^{28}$. If ice and rock are

165 homogeneously distributed throughout the body, and the body heats homogeneously, then large-

166 scale, coherent magnetization could exist. The length scale of magnetization would represent that

167 of any heterogeneous distribution of water ice, which would cause heterogeneity in the amount

168 of radiogenic heating from the silicate component. 
171 CAIs would undergo enough heating to acquire a CRM during aqueous alteration while

172 embedded within the solar nebular field, but not too much heating that would later erase the

173 CRM. Additionally, our work establishes an upper bound on the magnetic dipole moment that is

174 possible for a planetesimal magnetized by the solar nebula. We predict that large C-type

175 asteroids that exhibit evidence of partial differentiation could have magnetospheres detectable

176 from spacecraft. We hypothesize that Pallas is one such asteroid and show that this hypothesis

177 can be tested with low-altitude, magnetic field measurements. 

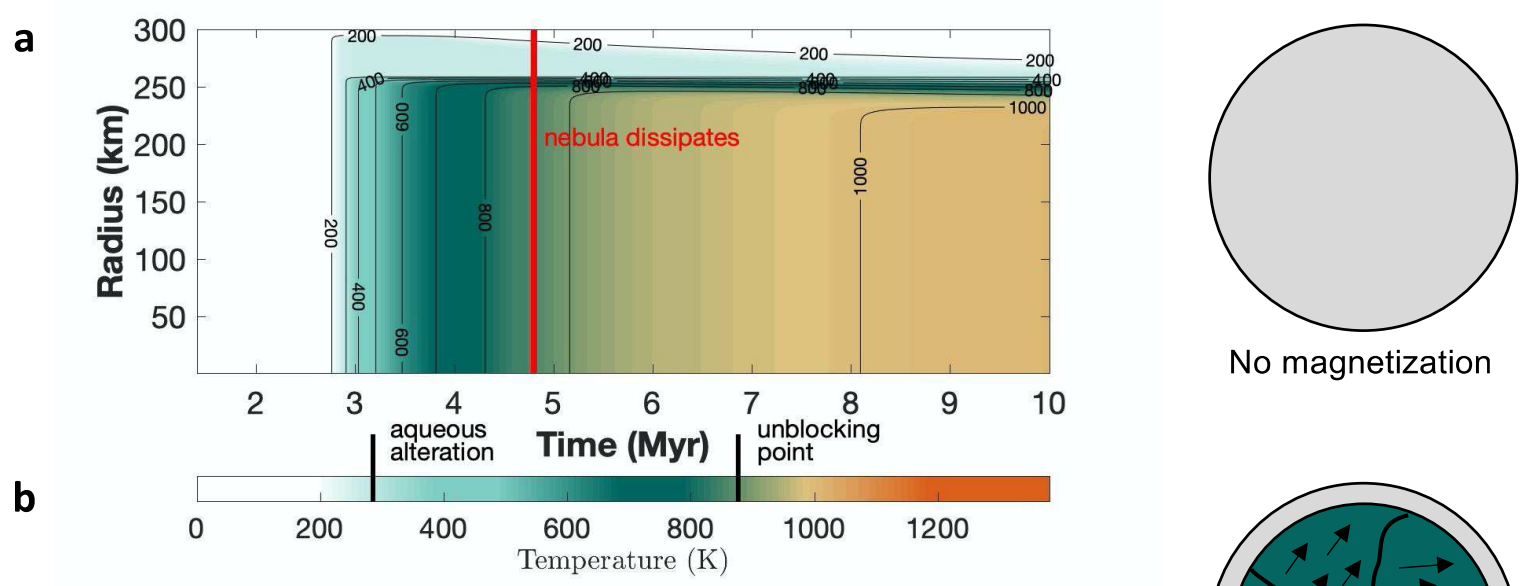

No magnetization

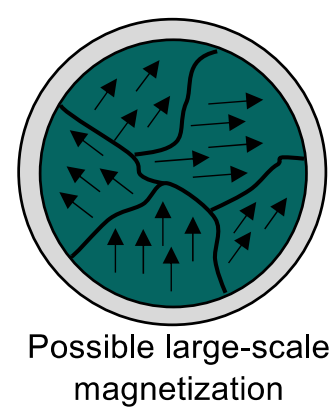

C
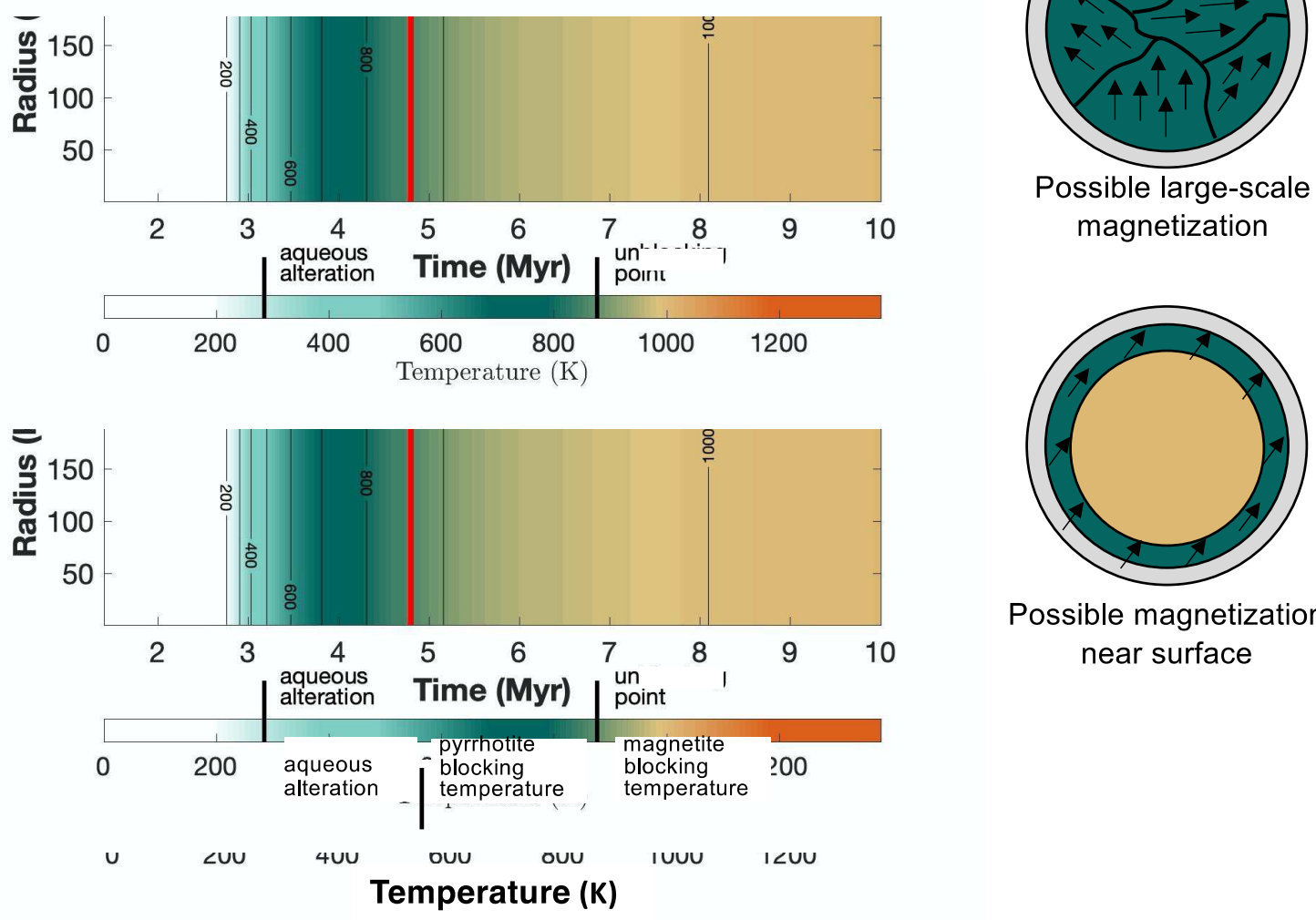

Possible magnetization near surface

181 Fig. 1: Planetesimal thermal evolution determines extent of aqueous alteration and thus

182 magnetizable volume. Thermal evolution models for a 300-km-radius planetesimal assuming

183 formation at (a) $4 \mathrm{Myr}$, (b) 3.2 Myr, and (c) 2.8 Myr after CAIs. In panel (a), the planetesimal

184 forms too late for ${ }^{26} \mathrm{Al}$-driven heating to trigger aqueous alteration before the solar nebular field

185 dissipates. This case leads to no magnetization. In panel (b), the planetesimal forms early enough 
186 for aqueous alteration to occur but does not subsequently exceed the unblocking temperatures of

187 the magnetic minerals. This scenario leads to possible coherent magnetization throughout the

188 entire body. In panel (c), the planetesimal heats enough to experience aqueous alteration before

189 the nebular field dissipates, but subsequently exceeds the unblocking temperature for most of the

190 interior. This evolution means that the majority of the planetesimal would be demagnetized

191 except for a thin shell near the surface.

192
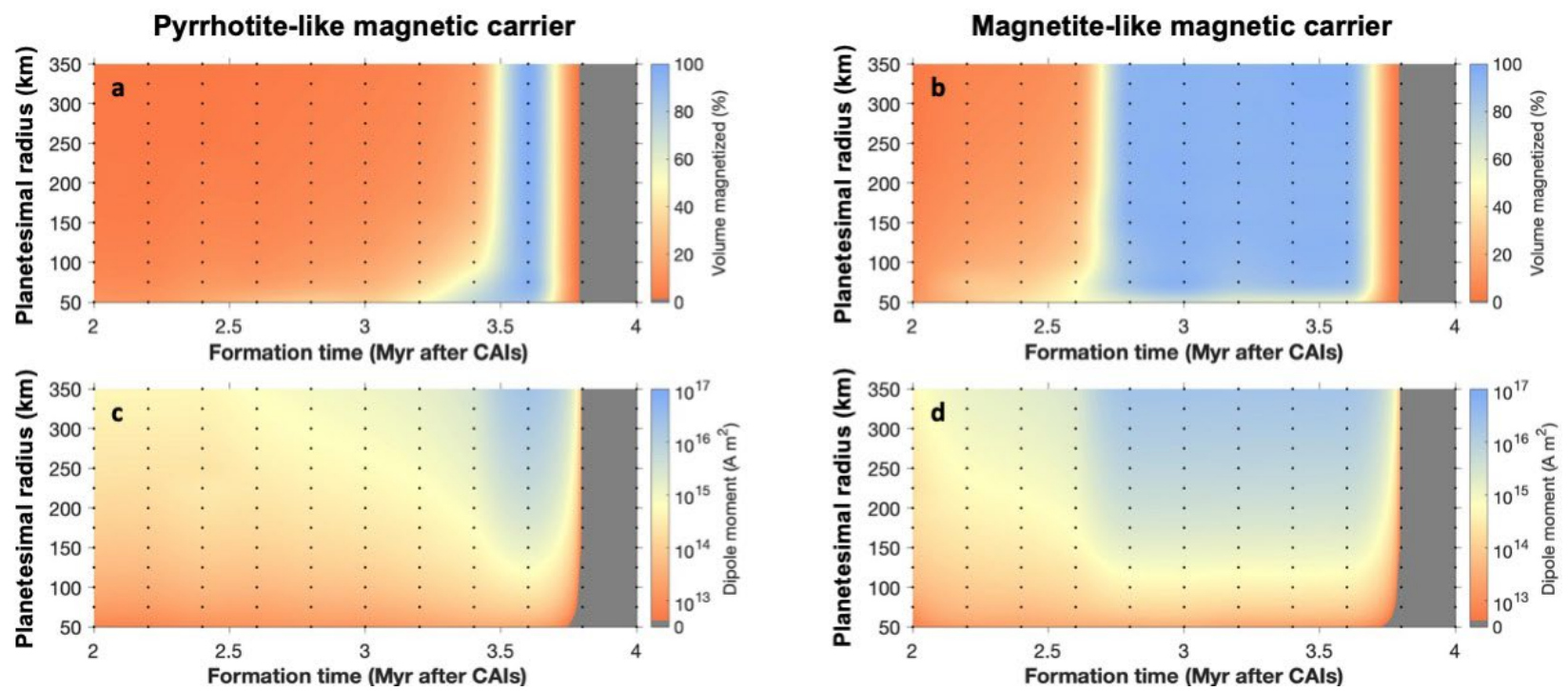

194 Fig. 2: Formation time determines the maximum possible magnetized rock volume and

195 magnetic moment that a planetesimal could acquire. Panels (a) and (b) illustrate the

196 maximum possible volume of the planetesimal that the solar nebular field could magnetize as a

197 function of the planetesimal's formation time and radius, assuming pyrrhotite and magnetite are

198 the magnetic carriers, respectively. A wide range of formation times lead to $\sim 100 \%$

199 magnetization if the magnetic carrier is magnetite. If the magnetic carrier is pyrrhotite, then the

200 extent of magnetization is more sensitive to formation time. Black dots represent individual

201 thermal model runs. We assume that the solar nebular field dissipated at 4.8 Myr after CAIs and 
202 that the unblocking temperature is $550 \mathrm{~K}$ for pyrrhotite and $850 \mathrm{~K}$ for magnetite. The greater the 203 volume percentage of a planetesimal that is magnetized, the greater its magnetic dipole moment 204 could be. Panels (c) and (d) illustrate the maximum possible dipole moment where the magnetic 205 carrier is pyrrhotite and magnetite, respectively. Here we use a magnetization intensity of $10^{-4} \mathrm{~A}$ $206 \mathrm{~m}^{2} \mathrm{~kg}^{-1}$.

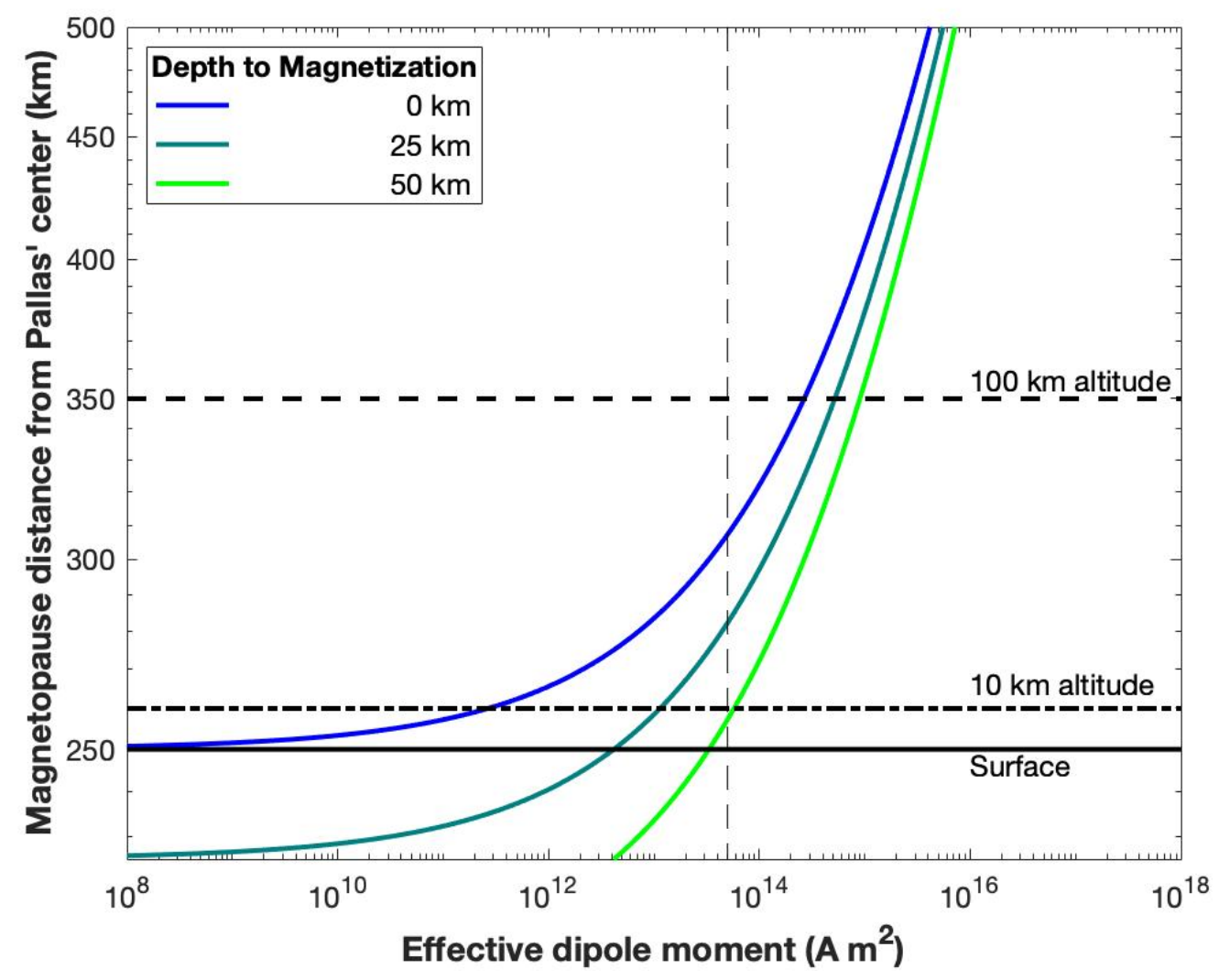

211 Fig. 3: A magnetized chondritic asteroid can produce a detectable magnetopause. The

212 curves represent the magnetopause distance as a function of a Pallas-sized asteroid's effective

213 magnetic dipole moment. The higher the magnetopause, the more easily detectable the asteroid's

214 magnetosphere. At low magnetic moments and close proximity to the asteroid's surface, the

215 magnetopause distance depends heavily on the depth to magnetization, with deeper 
216 magnetization being more difficult to detect. Moments greater than $\sim 10^{13}$ to $10^{14} \mathrm{~A} \mathrm{~m} \mathrm{~m}^{2}$ are likely

217 detectable during an orbiter or flyby. Lower magnetic moments could be detected by a low orbit

218 or a lander. The exact cutoffs for detectability depend on specific mission parameters.

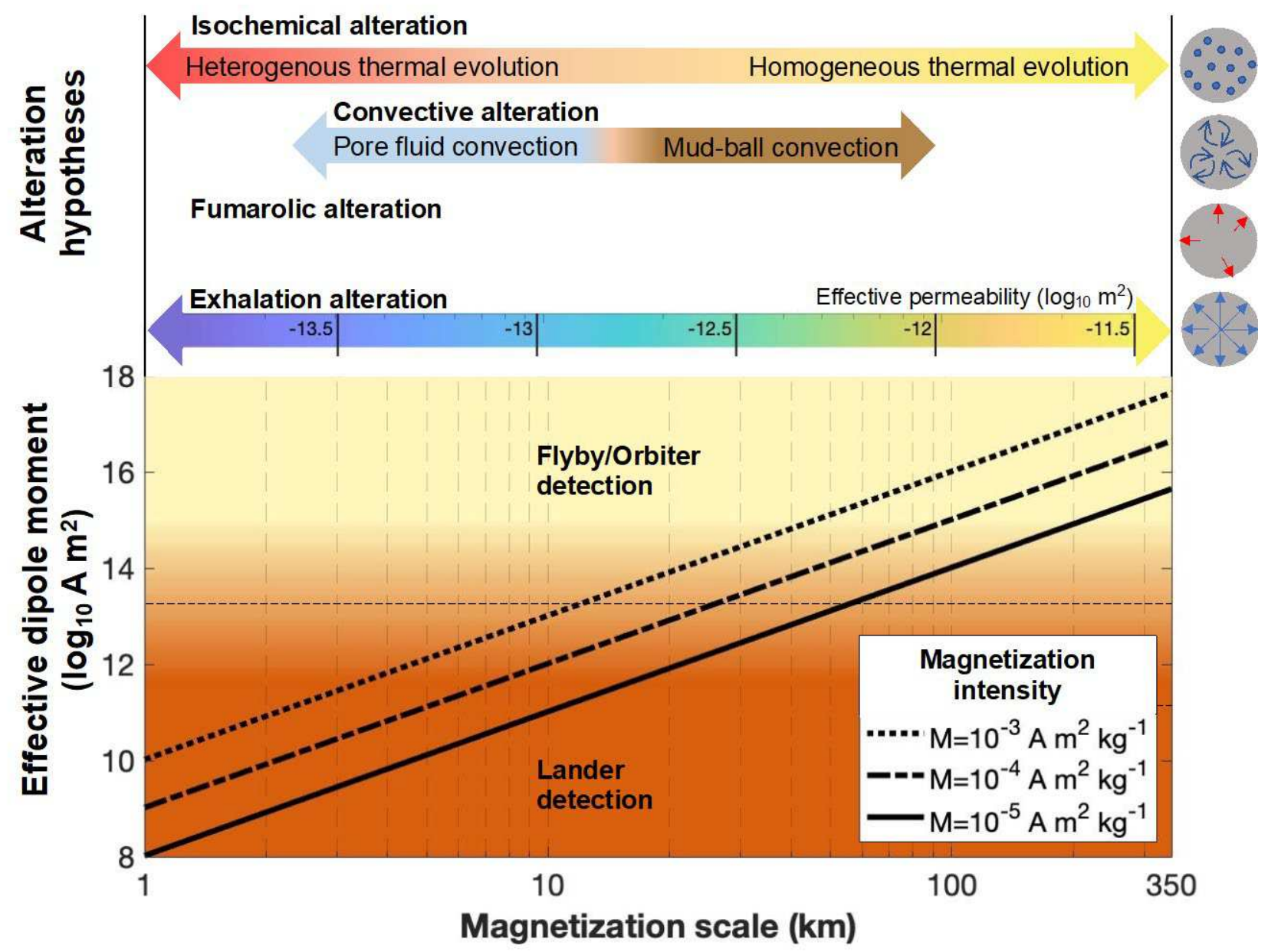

221 Fig. 4: The aqueous alteration model determines the length scale of coherent remanent

222 magnetization, which determines the magnetic dipole moment. We use three different

223 magnetization values corresponding to the range observed in CM chondrites ${ }^{3}$. The prevailing

224 aqueous alteration process determines the magnetization scale, which is the effective radius of

225 the volume that is coherently magnetized. Many aqueous alteration models exist. For outward 
226 Darcy fluid flow (exhalation flow), effective permeability controls the magnetization length

227 scale. If hydrothermal or fluid convection models control alteration, then the system or

228 convection scale likely determines the magnetization scale. Lastly, alteration may not depend on

229 fluid flow. In an isochemical model, the magnetization scale would depend on the homogeneity

230 of the ice/rock ratio that controls thermal evolution. From Fig. 3, we expect that the lowest

231 detectable dipole moment for an orbiter or flyby over the surface of Pallas is $\sim 10^{13.5} \mathrm{~A} \mathrm{~m}^{2}$. Thus,

232 it would be possible to detect magnetization from a spacecraft at Pallas if coherent magnetization

233 exists at a scale greater than $\sim 10-50 \mathrm{~km}$.

\section{Acknowledgements:}

236 We thank Dr. Alan Rubin for fruitful discussion about the alteration of CM chondrites. BPW and

237 RO thank the NASA Discovery Program (NNM16AA09 C) for support. Part of this work was

238 carried out at the Jet Propulsion Laboratory, California Institute of Technology, under a contract

239 with the National Aeronautics and Space Administration (80NM0018D0004). 
241 Thermal evolution models:

Thermal evolution controls how and when a planetesimal may acquire chemical remanent

243 magnetization through aqueous alteration. We employ the thermal modeling algorithm described

244 in Castillo-Rogez et al. $(2007,2010)^{29-31}$. This algorithm solves for the temperature profile at

245 each radial extent,

246

$$
\frac{d\left(\frac{k(T) d T(r)}{d r}\right)}{d r}+\frac{2}{r}\left(\frac{k(T) d T(r)}{d r}\right)=\rho(r) C_{p}(T)\left(\frac{d T(r)}{d t}\right)-H(r)
$$

247 where $T$ is the temperature, $r$ is the radius, $k$ is the thermal conductivity, $\rho$ is the density, $C_{p}$ is

248 the heat capacity, and $H$ is the specific heat production from radiogenic decay. We assume the 249 initial $\mathrm{Al}^{26}$ concentration is $600 \mathrm{ppb}$ and that its specific heat production is $0.146 \mathrm{~W} / \mathrm{kg}$. The 250 model time step is $10^{4}$ years. Additionally, this thermal model includes heating from accretion,

$$
T(r)=\frac{h_{a}}{C_{p}(T)}\left[\frac{4 \pi}{3} \rho G r^{2}+\frac{\langle v\rangle^{2}}{2}\right]+T_{i}
$$

252 where $h_{a}$ is the fraction of mechanical energy transferred to heat, $G$ is the gravitational constant,

$253 v$ is the velocity of accreting objects, and $T_{i}$ is the initial temperature. Even for the largest

254 planetesimals we consider, the heat from accretion is only a few 10 s of $\mathrm{K}$, so the assumption of

255 instantaneous accretion is acceptable. Full details of the model implementation, including the

256 assumed values of all the parameters, are available in Castillo-Rogez et al. $(2007)^{31}$.

We model an array of planetesimals with varying radii and formation time. Radii range

259 from $50-350 \mathrm{~km}$ in increments of $25 \mathrm{~km}$ and formation times range from 2-4 Myr after CAIs in 
261 of 10 and $40 \%$. We also run each model with initial temperatures of 100 and $160 \mathrm{~K}$, which

262 correspond to the expected outer (between the orbits of Jupiter and Uranus) and inner solar

263 system temperatures respectively.

264

265 Dipole moment calculation:

266 The magnetic dipole moment is defined as,

$$
m=\iiint \boldsymbol{M} d V,
$$

268 where $\boldsymbol{M}$ is the magnetization and $V$ is the magnetized volume. In general, an asteroid can be

269 non-spherical, and the volume could be composed of fractions that have varying magnetization

270 strengths and directions. To calculate the maximum possible dipole moment, we assume that the

271 entire magnetized volume of the asteroid has the same magnetization direction. Additionally, we

272 assume a radially symmetric asteroid. The dipole moment then simplifies to the product of the

273 magnetization magnitude and the magnetized volume,

$$
m=M V .
$$

275 The output of a planetesimal thermal model tells us what volume of the planetesimal can

276 be magnetized. We assume that the magnetization value is $10^{-4} \mathrm{~A} \mathrm{~m}^{2} / \mathrm{kg}$, which falls within the

277 range of values observed in $\mathrm{CM}$ chondrites ${ }^{3}$. One could attempt to calculate the magnetization of

278 the planetesimal by assuming a nebular field strength and orientation, without relying on any

279 meteoritic data. However, none of these parameters are constrained enough to provide a more 280 precise estimate than our preferred method ${ }^{32,33}$.

281 Research suggests that the intensity and direction of the solar nebular field was likely

282 stable for periods of $\sim 1 \mathrm{kyr}$ or longer ${ }^{1}$ - long enough to allow magnetic minerals to form during 283 a planetesimal's aqueous alteration ${ }^{34}$. However, a rotating planetesimal orbiting around the early 
284 Sun would have had an ever-changing orientation with respect to the nebular field. In this case,

285 the planetesimal would acquire remanent magnetization in the net average direction of the

286 background field parallel to the planetesimal's spin axis $^{35}$. If the planetesimal's spin axis is not

287 perfectly aligned with the disk plane, the projection of the spin axis onto the vertical component

288 of the nebular field in the protoplanetary disk would have a non-zero average, as the vertical

289 component would remain constant as the planetesimal orbits around the sun.

290 It is idealistic to assume that the solar nebula would magnetize an entire asteroid

291 unidirectionally. If fluid flow from the core to the surface of a planetesimal drives the rate at

292 which aqueous alteration occurs, the permeability of the planetesimal determines the length scale

293 of magnetization. Under the assumption of Darcy fluid flow, the pressure gradient in the body

294 drives fluid to the surface. The pressure gradient at a depth $z$ is defined as,

295

$$
\frac{\mathrm{dP}}{\mathrm{dz}}=\rho g(z)
$$

296 where $\rho$ is density, and $g$ is gravitational acceleration. The linear fluid velocity in a porous

297 medium governed by Darcy's law follows the relationship,

$$
v(z)=\frac{k}{\mu \phi} \frac{\mathrm{dP}}{\mathrm{dz}}
$$

299 where $k$ is permeability, $\mu$ is fluid viscosity, and $\phi$ is porosity. Dividing the expected stability

300 time of the nebular field, $\sim 1 \mathrm{kyr}$, by the velocity $v$, yields an estimate of the magnetization length

301 scale. We report the length scale using the maximum fluid flow velocity within the body. The

302 fluid viscosity of water is $10^{-3} \mathrm{~Pa}$ s. Assuming the length scale corresponds to the radius of a

303 spherical volume, the dipole moment is given by the product of the spherical volume and the 304 magnetization value. At a spacecraft flyby distance (i.e., closest approach of $\sim 100 \mathrm{~km}$ or less 305 from the surface of the planetesimal, depending on the mission design), a collection of randomly 
oriented magnetized regions of similar size would produce a field strength that is approximately

307 the same field strength as would come from the dipole moment of a single magnetized region.

309 Magnetopause distance:

310 To assess the detectability of a magnetized Pallas, we estimate the magnetopause distance. The

311 magnetopause distance is the point at which the solar wind pressure and the asteroid's magnetic

312 field pressure balance each other.

$$
D=R_{p}\left(\frac{f^{2} B^{2}}{2 \mu_{0} m_{H} n V^{2}}\right)^{\frac{1}{6}}
$$

314 where $R_{p}$ is the radius of the body, $m_{H}$ is the mass of a hydrogen ion, $n=2 \mathrm{~cm}^{-3}$ is the density of

315 the solar wind at the asteroid belt, $V=400 \mathrm{~km} / \mathrm{s}$ is the solar wind velocity, and $f=2$ is a

316 geometric factor ${ }^{36} . B$ is the field strength on the magnetic equator on the surface. For a magnetic

317 dipole, the field strength is

$$
B=\frac{\mu_{0}}{2 \pi} \frac{m}{R_{m}^{3}}
$$

319 Where $m$ is the magnetic moment, and $R_{m}$ is the distance from the dipole center. This equation

320 approximates the body's magnetic field as a dipole centered in the body. This approximation is

321 valid for strong magnetic moments and magnetopause distances that are far from the surface of

322 the body. However, small dipole moments as generated by groups of small length-scale dipoles

323 would have a more complicated behavior at close distances. In this case, the surface field would

324 be most dominated by the dipole that is closest. Thus, we plot the magnetopause distance

325 assuming that the distance from the dipole center to the surface is the same as the length scale of

326 the magnetization. 


\section{Supplementary information:}

\section{Simulation assumptions and parameter uncertainties}

Figure 2 applies to planetesimals that formed in the outer solar system where the nebula

331 dissipated $^{1}$ at a given time, in this case, 4.8 Myr. If the nebula dissipated sooner, then the range

332 in formation times that could lead to large-scale magnetization would be narrower. Alternatively,

333 a planetesimal is more likely to retain a CRM if the nebula dissipated later. Additionally, our

334 thermal models assume that the planetesimals contain 40 vol\% water ice. A lower water-to-rock

335 ratio or higher concentration of ${ }^{26} \mathrm{Al}$ in the rock fraction would lead to faster heating and a

336 narrower range of formation times that could result in large-scale, magnetization. If heating is

337 sufficiently higher than our nominal scenario, large-scale magnetism dominated by pyrrhotite is

338 not possible because a planetesimal would always exceed pyrrhotite's unblocking temperature.

339 Extended Data Figs. 1 and 2 illustrate the effects of quicker nebular dissipation and a lower

340 water-to-rock ratio, respectively.

The process of aqueous alteration occurs when water ice melts at $273 \mathrm{~K}$. This assumes

343 the ice fraction is pure water ice. In our thermal models, differentiation occurs instantaneously ${ }^{37}$.

344 This assumption is valid because the timescale of water transport is likely much shorter than the

345 timescale of the thermal evolution ${ }^{30,31}$. When the model reaches the water melting point, the

346 water migrates toward the surface of the planetesimal. This time marks the point at which

347 aqueous alteration occurs throughout the planetesimal. Our results are largely independent of

348 planetesimal size for radii greater than $\sim 50 \mathrm{~km}$. For smaller planetesimals, the amount of

349 volumetric radiogenic heat generated versus heat lost radiating from the surface becomes an

350 important factor. However, the thermal models are only accurate for diameters greater than 
351 approximately $50 \mathrm{~km}$; below this diameter, the assumption of instantaneous differentiation

352 leading to the migration of liquid water to the surface is no longer valid. The models are

353 computed accurately under the assumption that the differentiated body has a rocky mantle and

354 water layer. From a thermal evolution standpoint, these models are upper bounds on the max 355 temperature achieved.

358 material (i.e., no alteration during accretion) and that no disruption occurred to the planetesimal

359 during its thermal evolution. Although C-type asteroids could have accreted material that was

360 already aqueous altered to begin with ${ }^{14}$, this assumption would not alter our conclusions so long

361 as additional alteration could take place. The assumption of instantaneous accretion is acceptable

362 because under the paradigm of pebble accretion, planetesimals likely formed quickly relative to

363 the timescale of radiogenic heating ${ }^{38}$. Instabilities in the protoplanetary disc could bring together

364 bodies on the order of $10 \mathrm{~s}-100 \mathrm{~s}$ of $\mathrm{km}$ over $\mathrm{kyrs}^{33}$. Indeed, the theory of planet formation by

365 pebble accretion requires a large reservoir of planetesimals of this size which could subsequently

366 grow into protoplanets by continuing aggregation of dust and pebbles. However, alteration

367 during protracted accretion or catastrophic disruptions may still have occurred which we do not 368 consider $^{39}$.

\section{Alternative origins of magnetization in planetesimals}


374 planetesimal once hosted a dynamo, presumably in a metallic core that is vigorously convecting

375 due to rapid cooling. Therefore, a planetesimal can only host a dynamo if it heated up enough to

376 melt metal and form a liquid core. Chondrites are undifferentiated material; however, they may

377 originate from the outermost shells of parent bodies that are internally differentiated. That is,

378 some chondritic material may remain relatively cold near the surface of a differentiated body $y^{40,41}$

379 but experience an endogenous magnetic field from a core dynamo in the hot interior ${ }^{42}$. In other

380 words, steep thermal gradients in the outermost layers of a parent body could keep the exterior

381 cool even if the interior is relatively hot.

Formation of a metallic cores can occur as early as a few Myr after CAIs for bodies that

384 formed early in the history of the solar system with scant water ice ${ }^{40}$. However, studies of 385 asteroid dynamos suggest that the onset of a magnetic field would not occur until $\sim 5 \mathrm{Myr}$ after 386 CAIs or later ${ }^{40,42}$ because the core needs to start cooling to kickstart a dynamo. Radiogenic 387 dating of carbonate minerals associated with aqueous alteration in $\mathrm{CM}$ chondrites places their 388 formation time between $\sim 2.4$ and $4 \mathrm{Myr}$ after CAIs ${ }^{43}$. Based on alteration sequences proposed for $389 \mathrm{CM}$ chondrites, these minerals likely formed contemporaneously with pyrrhotite and 390 magnetite ${ }^{2,44}$. Thus, it is unlikely that an endogenous magnetic field from a core dynamo 391 magnetized the $\mathrm{CM}$ chondrites if model-predicted dynamo timing and the ages of the $\mathrm{CM}$ 392 magnetized minerals are correct. A spacecraft detection of a magnetosphere around Pallas would add evidence to the 395 hypothesis that Pallas is a possible parent body for the CM chondrites, assuming that the 396 spacecraft could also rule out the presence of a large metallic core. The absence of a metallic 
397 core would rule out a dynamo origin for the magnetization. Measurements of the density of the

398 near-surface material, compared to the bulk density and (if available) the moment of inertia,

399 would help determine the extent of internal differentiation. Knowledge of the internal

400 differentiation state could constrain whether a metallic core may exist to host a dynamo source ${ }^{42}$.

401 Additionally, the magnetization could not be confused with accretional-detrital-remanent

402 magnetization ${ }^{45}$. Accretional-detrital-remanent magnetization is produced by magnetized grains

403 aligning themselves during the process of accretion in the solar nebular field. However, this

404 magnetization process would not produce coherent magnetization above the meter-scale ${ }^{45}$, and

405 thus only a magnetometer landed on the surface of an asteroid could detect accretional-detrital-

406 remanent magnetization. Thus, observations of large-scale magnetization on Pallas combined

407 with the absence of a core would uniquely confirm that it formed before the solar nebular

408 dissipated.

409

410 In principle, a strong solar wind - as opposed to the solar nebular field — could magnetize

411 parts of planetesimals. Although the early solar wind was stronger than the solar wind today, it

412 was probably still too weak to magnetize chondritic material ${ }^{35}$. Nevertheless, one recent study ${ }^{46}$

413 suggested that the early solar wind could magnetize planetesimals because young planetesimals

414 would behave more like comets and have conductive outer shells that could amplify the

415 magnetizing field by an order of magnitude near the surface. In this scenario, CM chondrites

416 could originate from the magnetized outer shell of a planetesimal. Whether magnetization by the

417 solar wind could produce a planetesimal with a magnetized shell remains controversial ${ }^{35}$. In any

418 case, the solar wind is not expected to produce planetesimal with large-scale magnetization. 
419 Again, observations of a strong remanent magnetization could reveal the timing of the formation

420 of planetesimals like Pallas.

421

\section{Length scale of magnetization}

423 The rate of fluid flow in planetesimals could have controlled the rate of mineral

424 alteration ${ }^{23}$. In this case, the greater the permeability of the planetesimal, the greater the length

425 scale of magnetization. The permeability of a planetesimal at early times is difficult to determine.

426 Estimates ${ }^{25,28}$ based on geodynamical arguments and/or measurements of meteorites and

427 terrestrial analogs range widely from $\sim 10^{-19}$ to $10^{-11} \mathrm{~m}^{2}$. Measurements of permeability in

428 meteorite samples tend to favor low permeability values ${ }^{28,47}$; however, geodynamical models of

429 aqueous alteration often assume high permeability ${ }^{25,48}$. These need not be in complete agreement.

430 Although the permeability of a meteorite hand sample is low, the overall effective permeability

431 of a planetesimal could be much greater due to large fractures and fluid conduits ${ }^{24}$ or because the

432 material has not yet been lithified ${ }^{23}$. If the effective permeability is high, then fluid could flow

433 quickly from the planetesimal's interior to its surface in kyrs, i.e., exhalation flow ${ }^{49}$. Quick

434 exhalation flow could produce large-scale, coherent magnetization of potentially the entire

435 planetesimal body. Per contra, fast exhalation flow might not allow enough time for chemical

436 reactions to occur ${ }^{49}$. Multiple pass fluid flow, e.g., pore fluid convection, may be required to

437 produce the degree of aqueous alteration seen in $\mathrm{CM}$ chondrites ${ }^{50}$. Near the surface of a

438 planetesimal, fluid flow could be dominated by convection ${ }^{25}$. The thermal gradient near the

439 surface would cause warm water to rise while cold water descends. Under this regime, we would

440 expect the magnetization length scale would be approximately the convection length scale.

441 Modeling of pore fluid convection indicates scales of $\sim 10 \mathrm{~km}$. Lastly, high temperature 
442 hydrothermal systems may occur within planetesimals ${ }^{51}$. High temperature hydrothermal

443 reactions in fumarolic-like systems that involve effusive events of steam are potentially able to

444 alter minerals much more quickly (10s of yrs or less) than conventional fluid alteration ${ }^{34}$.

445 However, fumarolic alteration would be limited to small, localized regions. From terrestrial 446 analogs ${ }^{52}$, we expect this length scale would be on the order of $1-10 \mathrm{~km}$.

If the permeability of a planetesimal is as low as $10^{-19} \mathrm{~m}^{2}$, then fluid flow would be so

449 slow that effectively all aqueous alteration would occur with stagnant and chemically isolated 450 water $^{28}$. This model matches geochemical studies of CM chondrites which indicate alteration

451 occurred isochemically ${ }^{25,28}$. In the previous paragraph, we implicitly assume that the length scale

452 of magnetization would be determined by the distance that fluid can travel over the period of 453 nebular field stability ( $\sim 1 \mathrm{kyr})$. However, aqueous alteration that occurs from chemically isolated

454 fluids would produce magnetization length scales that depend on the homogeneity of internal 455 heating. If water ice is distributed homogeneously throughout the planetesimal's body, then we 456 would expect most of the interior to reach the ice melting point around the same time. At this 457 point, all rock would be exposed to at least a little water which may or may not be trapped.

458 Under this assumption, the magnetization length scale then depends on how homogenously the

459 body heats, which is dependent on how homogeneously the water ice and radiogenic isotopes are 460 distributed. In the absence of constraints, this could lead to any length scale value.

Finally, debates over permeability and whether alteration was isochemical can be

463 bypassed altogether if aqueous alteration occurred in un-lithified rock. In this case, water melting 464 leads to a muddy mixture of mobile pebbles ${ }^{27}$. Since both the pebbles and the fluid are well 
465 mixed and mobile, a muddy planetesimal may experience "mudball" convection, wherein both

466 solids and fluid move about the asteroid in order to dissipate heating ${ }^{27,53}$. This whole-body

467 convection can extend from the mud-mantle to the core. The sorting of particle sizes and variable

468 water-to-rock ratio throughout a convecting mudball would lead to compositional heterogeneity

469 over short length scales ${ }^{27}$. We expect that magnetization length scales would be on the order of

$470 \sim 10-100 \mathrm{~km}$ based on the size of convective cells.

471

472 To summarize, if exhalation flow dominates the magnetic length scale, then the scale

473 would be determined by the permeability of the body. Under the assumption of permeable

474 material, we expect magnetization scales to be on the order of 10-100 km. Under the realm of

475 fluid convection dominated alteration, we expect that only the near surface within kms of the

476 surface would have the temperature gradient necessary to sustain convection, so length scales

477 would be $\sim 10 \mathrm{~km}$. If fumarolic systems dominate magnetization, then based on terrestrial

478 hydrothermal scales, the length scale could be on the order of $1-10 \mathrm{~km}$. If the asteroid is not

479 permeable, then alteration scales could take on a wide range of values depending on the

480 homogeneity of temperature increase throughout the body (e.g., from $<1 \mathrm{~km}$ to $>100 \mathrm{~km}$ ).

481 Finally, "mudball" convection could lead to magnetization length scales of up to $100 \mathrm{~km}$.

\section{Constraints from spacecraft observations}

484 To date, only one spacecraft with a magnetometer has visited a C-Type asteroid. The

485 MASCOT lander aboard JAXA's Hayabusa2 mission made magnetic field measurements on the

486 surface of the C-type, rubble-pile asteroid (162173) Ryugu ${ }^{54}$. However, the MASCOT lander did

487 not detect a magnetic field intrinsic to Ryugu ${ }^{54}$. This means that if Ryugu has magnetization, it 
488 must be at a scale less than $1 \mathrm{~cm}^{54}$. This does not preclude the possibility of magnetized C-type 489 asteroids. First, Ryugu is a rubble pile, so strong coherent magnetization is not possible as any 490 magnetized pebbles or boulder would have scrambled orientations. Furthermore, Ryugu's

491 composition may not match that of aqueously altered chondrites, and thus are not applicable to 492 this study. However, if samples from Ryugu do match CM chondrites for example, and do not 493 have any detectable magnetization or evidence that magnetization could have been erased, then 494 this would suggest that magnetization of CM chondrites is not a ubiquitous process occurring 495 throughout the entire body of an original CM chondrite parent body. Preliminary publications 496 suggest Ryugu is more like CI chondrites ${ }^{55}$.

498 Although NASA's Dawn spacecraft did not have a magnetometer, Dawn did visit the 499 largest C-type asteroid, 1 Ceres. Using observations from the Gamma Ray and Neutron Detector 500 (GRaND), one can place an upper limit on Ceres' magnetic field by analyzing the deflection of 501 charged particles ${ }^{56}$. Although GRaND made transient detections of deflected particles, it did not 502 observe a signal consistent with a bow shock from a strong dipolar field in the solar wind ${ }^{56}$.

503 Through this argument, Ceres cannot have a magnetic moment greater than $\sim 10^{16} \mathrm{~A} \mathrm{~m}^{2}$ or so $^{56}$.

504 This limit does not rule out large-scale, coherent magnetization, so we cannot exclude the 505 possibility that Ceres has a detectable CRM from the solar nebular field. 


\section{Extended Data Figures:}
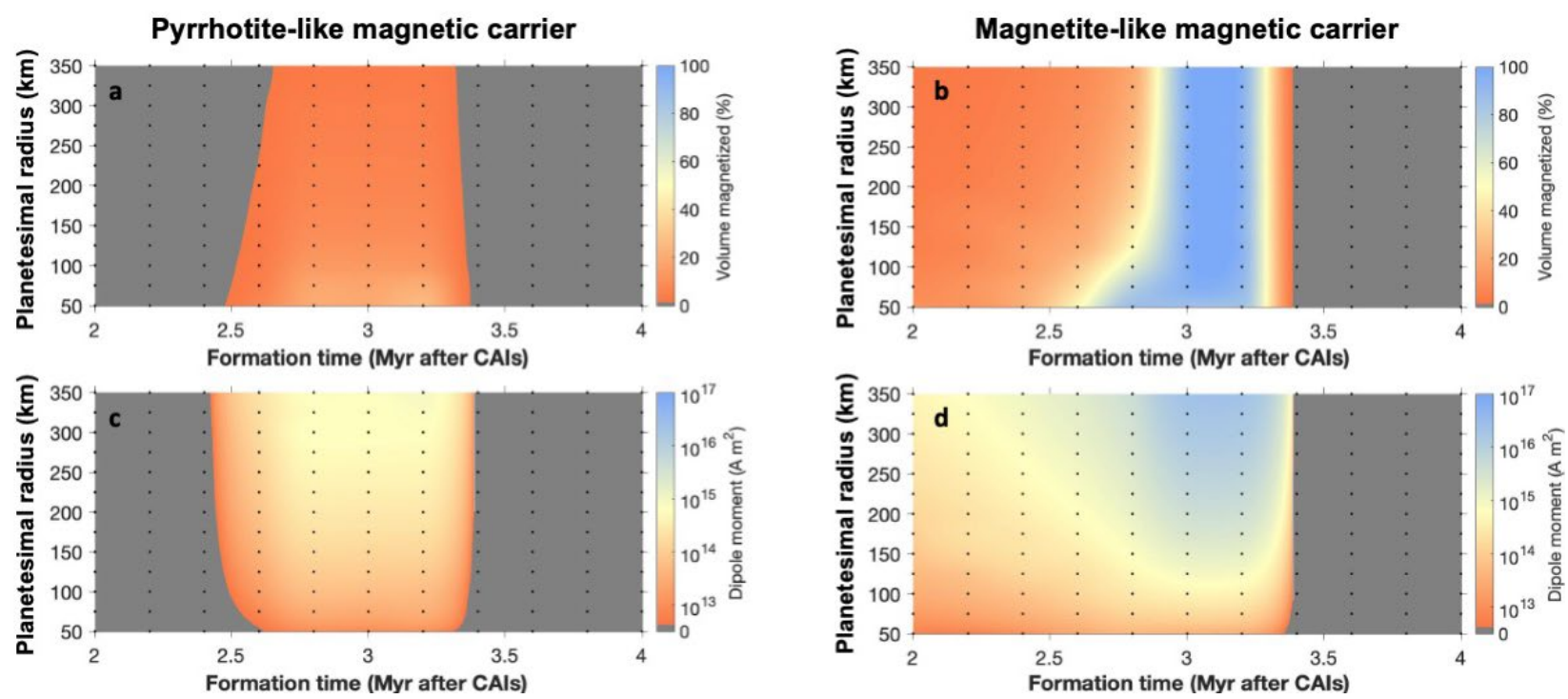

509 Extended Data Figure 1: Maximum magnetized volume percent for planetesimals with 40 vol\%

510 water ice that formed in the inner solar system where the solar nebula dissipated at 3.9 Myr after

511 CAIs and assuming an unblocking temperature of (a) $550 \mathrm{~K}$ and (b) $850 \mathrm{~K}$, which correspond to

512 the magnetic carriers pyrrhotite and magnetite respectively. Panels (c) and (d) illustrate the

513 maximum possible dipole moment corresponding to panels (a) and (b) respectively. Quicker

514 nebula dissipation leads to fewer planetesimals that could be magnetized. Compared to the

515 nominal case in Fig. 2, the range of time that can lead to $100 \%$ magnetization has been greatly

516 reduced if magnetite is the carrier and eliminated if pyrrhotite is the carrier. 

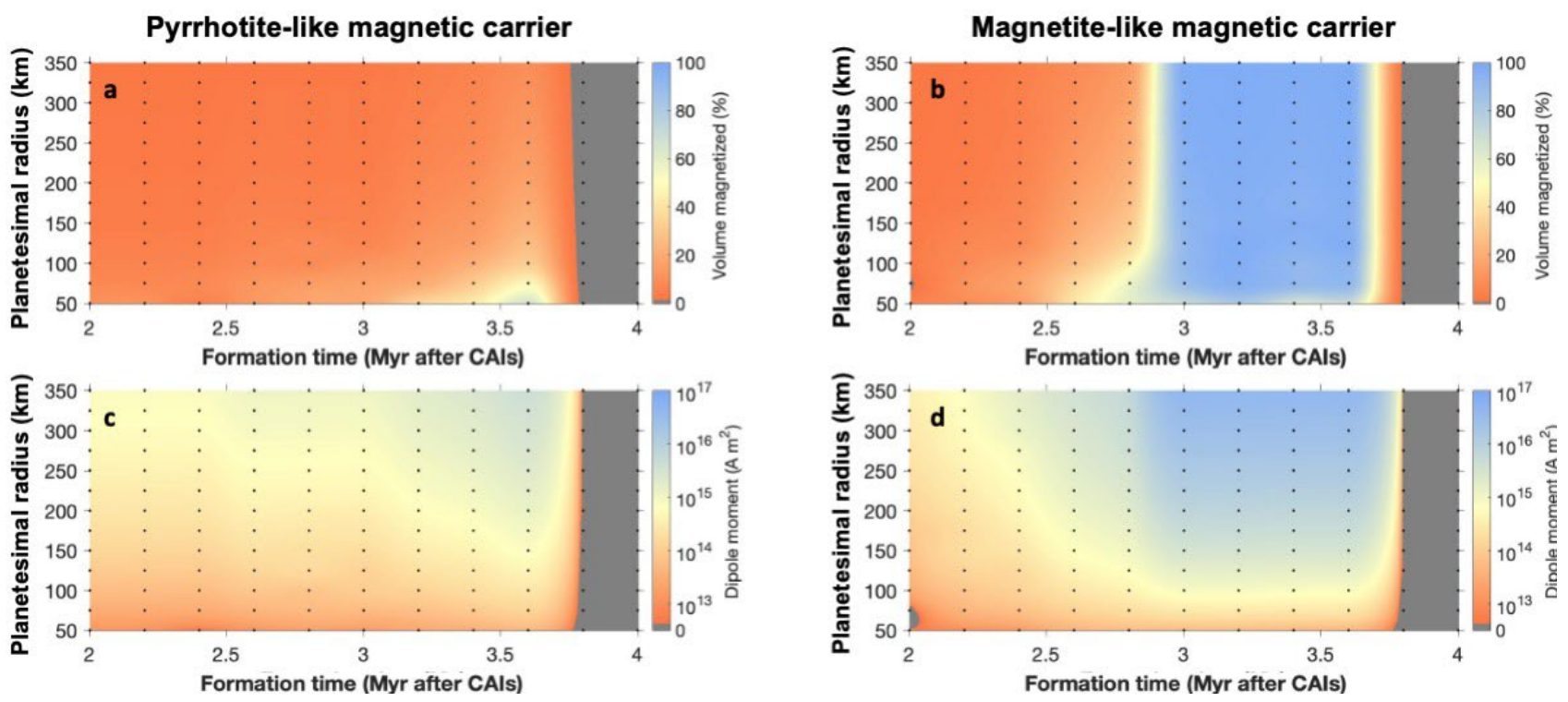

518 Extended Data Figure 2: Maximum magnetized volume percent for planetesimals with 10 vol\%

519 water ice that formed in the outer solar system where the solar nebula dissipated at 4.8 Myr after

520 CAIs and assuming an unblocking temperature of (a) $550 \mathrm{~K}$ and (b) $850 \mathrm{~K}$, which corresponds to

521 the magnetic carrier being pyrrhotite and magnetite respectively. Panels (c) and (d) illustrate the

522 maximum possible dipole moment corresponding to panels (a) and (b) respectively. Because

523 there is less water ice, there is more radiogenic heating. More radiogenic heating means it is

524 easier to reach the unblocking temperature(s) and erase magnetization. Compared to the nominal

525 case in Fig. 2, the range in time that allows for 100\% magnetization assuming magnetite is the

526 magnetic carrier has been narrowed. No times allow 100\% magnetization assuming pyrrhotite is

527 the carrier. A 10 vol\% water ice planetesimal would not have enough water to undergo complete

528 aqueous alteration, and low water content is not likely in the outer solar system. Furthermore,

529 even if it did occur, magnetite and pyrrhotite may not form, as hematite and tochilinite could be

530 the preferred minerals. Regardless, this scenario illustrates that models incorporating lower water

531 ice, or greater heating from a higher ${ }^{26} \mathrm{Al}$ abundance, are less favorable for large scale

532 magnetization. 


\section{References:}

535 1. Weiss, B. P., Bai, X.-N. \& Fu, R. R. History of the solar nebula from meteorite

536 paleomagnetism. Sci. Adv. 7, eaba5967 (2021).

537 2. Fu, R. R. et al. The Fine-Scale Magnetic History of the Allende Meteorite: Implications

538 for the Structure of the Solar Nebula. $A G U A d v .2,1-21$ (2021).

539 3. Cournede, C. et al. An early solar system magnetic field recorded in CM chondrites. Earth

$540 \quad$ Planet. Sci. Lett. 410, 62-74 (2015).

541 4. Rubin, A. E., Trigo-Rodríguez, J. M., Huber, H. \& Wasson, J. T. Progressive aqueous

542 alteration of CM carbonaceous chondrites. Geochim. Cosmochim. Acta 71, 2361-2382

$543 \quad$ (2007).

544 5. Marsset, M. et al. The violent collisional history of aqueously evolved (2) Pallas. Nat. 545 Astron. (2020) doi:10.1038/s41550-019-1007-5.

546 6. Carporzen, L. et al. Magnetic evidence for a partially differentiated carbonaceous

547 chondrite parent body. Proc. Natl. Acad. Sci. 108, 6386 LP - 6389 (2011).

548 7. Stacey, F. D. \& Banerjee, S. K. Chemical Remanent Magnetization (CRM). Part Vol. 549 Phys. Princ. Rock Magn. 128-135 (1974) doi:10.1016/b978-0-444-41084-9.50013-8.

550 8. Stacy, F. D. Paleomagnetism of meteorites. Annu. Rev. Earth Planet. Sci. 4, 147-157 $551 \quad(1976)$

552 9. Symington, N. H., Harries, T. J., Kurosawa, R. \& Naylor, T. T Tauri stellar magnetic 553 fields: He I measurements. Mon. Not. R. Astron. Soc. 358, 977-984 (2005).

554 10. Fu, R. R. et al. Solar nebula magnetic fields recorded in the Semarkona meteorite. Science $555 \quad$ (80-. ). 346, 1089-1092 (2014). 
556 11. Borlina, C. S. et al. Paleomagnetic evidence for a disk substructure in the early solar

$557 \quad$ system. Sci. Adv. 7, eabj6928 (2021).

558 12. Lowrie, W. Identification of ferromagnetic minerals in a rock by coercivity and

559 unblocking temperature properties. Geophys. Res. Lett. 17, 159-162 (1990).

560 13. Ciesla, F. J., Davison, T. M., Collins, G. S. \& O'Brien, D. P. Thermal consequences of 561 impacts in the early solar system. Meteorit. Planet. Sci. 48, 2559-2576 (2013).

562 14. Desch, S. J., Kalyaan, A. \& Alexander, C. M. O. The Effect of Jupiter's Formation on the 563 Distribution of Refractory Elements and Inclusions in Meteorites. Astrophys. J. Suppl. Ser. $564 \quad 238,11(2018)$.

565 15. Elkins-Tanton, L. T. et al. Observations, Meteorites, and Models: A Preflight Assessment 566 of the Composition and Formation of (16) Psyche. J. Geophys. Res. Planets 125, 1-23 567 (2020).

568 16. Larson, H. P., Feierberg, M. A. \& Lebofsky, L. A. The composition of asteroid 2 Pallas $569 \quad$ and its relation to primitive meteorites. Icarus 56, 398-408 (1983).

570 17. Macke, R. J., Consolmagno, G. J. \& Britt, D. T. Density, porosity, and magnetic 571 susceptibility of carbonaceous chondrites. Meteorit. Planet. Sci. 46, 1842-1862 (2011).

572 18. Schmidt, B. E. \& Castillo-Rogez, J. C. Water, heat, bombardment: The evolution and $573 \quad$ current state of (2) Pallas. Icarus 218, 478-488 (2012).

574 19. Carry, B. et al. Physical properties of (2) Pallas. Icarus 205, 460-472 (2010).

575 20. Blanco-Cano, X. \& Omidi, N. Hybrid simulations of solar wind interaction with 576 magnetized asteroids: Comparison with Galileo observations near Gaspra and Ida. $J$. 577 Geophys. Res. Sp. Phys. 108, 1-13 (2003).

578 21. Fatemi, S., Poppe, A. R., Delory, G. T. \& Farrell, W. M. AMITIS: A 3D GPU-Based 
Hybrid-PIC Model for Space and Plasma Physics. J. Phys. Conf. Ser. 837, (2017).

580

581

582

583

584

585

586

587

588

589

590

591

592

593

594

595

596

597

598

599

600

601

22. Unti, T. W. J. \& Neugebauer, M. Alfvén Waves in the Solar Wind. Phys. Fluids 11, 563568 (1968).

23. Young, E. D., Ash, R. D., Philip, E. \& Douglas, R. Fluid Flow in Chondritic Parent Bodies: Deciphering the Compositions of Planetesimals. Science (80-. ). 286, 1331-1335 (1999).

24. Kaplan, H. H. et al. Bright carbonate veins on asteroid (101955) Bennu: Implications for aqueous alteration history. Science (80-. ). 370, eabc3557 (2020).

25. Young, E. D., Zhang, K. K. \& Schubert, G. Conditions for pore water convection within carbonaceous chondrite parent bodies - implications for planetesimal size and heat production. Earth Planet. Sci. Lett. 213, 249-259 (2003).

26. Jamieson, J. W. et al. Sulfide geochronology along the Endeavour Segment of the Juan de Fuca Ridge. Geochemistry, Geophys. Geosystems 14, 2084-2099 (2013).

27. Bland, P. A. \& Travis, B. J. Giant convecting mud balls of the early solar system. Sci. $A d v .3, \mathrm{e} 1602514$ (2021).

28. Bland, P. A. et al. Why aqueous alteration in asteroids was isochemical: High porosity $\neq$ high permeability. Earth Planet. Sci. Lett. 287, 559-568 (2009).

29. Castillo-Rogez, J. C. \& Schmidt, B. E. Geophysical evolution of the Themis family parent body. Geophys. Res. Lett. 37, 1-5 (2010).

30. Castillo-Rogez, J. C. \& McCord, T. B. Ceres' evolution and present state constrained by shape data. Icarus 205, 443-459 (2010).

31. Castillo-Rogez, J. C. et al. Iapetus' geophysics: Rotation rate, shape, and equatorial ridge. Icarus 190, 179-202 (2007). 
602 32. Wardle, M. Magnetic fields in protoplanetary disks. Astrophys. Space Sci. 311, 35-45

$603 \quad(2007)$

604 33. Johansen, A. \& Lambrechts, M. Forming Planets via Pebble Accretion. Annu. Rev. Earth

$605 \quad$ Planet. Sci. 45, 359-387 (2017).

606 34. Ganino, C. \& Libourel, G. Fumarolic-like activity on carbonaceous chondrite parent body.

607 Sci. Adv. 6, eabb1166 (2020).

608 35. Oran, R., Weiss, B. P. \& Cohen, O. Were chondrites magnetized by the early solar wind?

609 Earth Planet. Sci. Lett. 492, 222-231 (2018).

610 36. Oran, R. et al. Maximum energies of trapped particles around magnetized planets and

$611 \quad$ small bodies. 1-23.

612 37. Sridhar, S., Bryson, J. F. J., King, A. J. \& Harrison, R. J. Constraints on the ice

613 composition of carbonaceous chondrites from their magnetic mineralogy. Earth Planet.

$614 \quad$ Sci. Lett. 576, 117243 (2021).

615 38. Morbidelli, A., Bottke, W. F., Nesvorný, D. \& Levison, H. F. Asteroids were born big.

$616 \quad$ Icarus 204, 558-573 (2009).

617 39. Maurel, C. et al. Meteorite evidence for partial differentiation and protracted accretion of

618 planetesimals. Sci. Adv. 6, 1-10 (2020).

619 40. Elkins-Tanton, L. T., Weiss, B. P. \& Zuber, M. T. Chondrites as samples of differentiated 620 planetesimals. Earth Planet. Sci. Lett. 305, 1-10 (2011).

621 41. Fu, R. R. \& Elkins-Tanton, L. T. The fate of magmas in planetesimals and the retention of

622 primitive chondritic crusts. Earth Planet. Sci. Lett. 390, 128-137 (2014).

623 42. Bryson, J. F. J., Neufeld, J. A. \& Nimmo, F. Constraints on asteroid magnetic field 624 evolution and the radii of meteorite parent bodies from thermal modelling. Earth Planet. 
Sci. Lett. 521, 68-78 (2019).

626 43. de Leuw, S., Rubin, A. E., Schmitt, A. K. \& Wasson, J. T. 53Mn-53Cr systematics of 627 carbonates in CM chondrites: Implications for the timing and duration of aqueous 628 alteration. Geochim. Cosmochim. Acta 73, 7433-7442 (2009).

629 44. Suttle, M. D., King, A. J., Schofield, P. F., Bates, H. \& Russell, S. S. The aqueous 630 alteration of CM chondrites, a review. Geochim. Cosmochim. Acta 299, 219-256 (2021).

631 45. Fu, R. R. \& Weiss, B. P. Detrital remanent magnetization in the solar nebula. J. Geophys. $632 \quad$ Res. Planets 117, (2012).

633 46. O'Brien, T. et al. Arrival and magnetization of carbonaceous chondrites in the asteroid 634 belt before 4562 million years ago. Commun. Earth Environ. 1, 1-7 (2020).

635 47. Corrigan, C. M. et al. The porosity and permeability of chondritic meteorites and 636 interplanetary dust particles. Meteorit. Planet. Sci. 32, 509-515 (1997).

637 48. Travis, B. J. \& Schubert, G. Hydrothermal convection in carbonaceous chondrite parent 638 bodies. Earth Planet. Sci. Lett. 240, 234-250 (2005).

639 49. Hutchison, R., Pillinger, C., Turner, G., Russell, S. \& Young, E. D. The hydrology of 640 carbonaceous chondrite parent bodies and the evolution of planet progenitors. Philos. 641 Trans. R. Soc. London. Ser. A Math. Phys. Eng. Sci. 359, 2095-2110 (2001).

642 50. Grimm, R. E. \& Mcsween, H. Y. Water and the thermal evolution of carbonaceous $643 \quad$ chondrite parent bodies. Icarus 82, 244-280 (1989).

644 51. Neveu, M., Desch, S. J. \& Castillo-Rogez, J. C. Core cracking and hydrothermal 645 circulation can profoundly affect Ceres' geophysical evolution. J. Geophys. Res. Planets $646 \quad \mathbf{1 2 0}, 123-154(2015)$.

647 52. Fujii, M., Sato, H., Togawa, E., Shimada, K. \& Ishibashi, J. Seafloor hydrothermal 
648 alteration affecting magnetic properties of abyssal basaltic rocks: insights from back-arc

$649 \quad$ lavas of the Okinawa Trough. Earth, Planets Sp. 70, 196 (2018).

650 53. Travis, B. J., Bland, P. A., Feldman, W. C. \& Sykes, M. V. Hydrothermal dynamics in a

651 CM-based model of Ceres. Meteorit. Planet. Sci. 53, 2008-2032 (2018).

652 54. Hercik, D. et al. Magnetic Properties of Asteroid (162173) Ryugu. J. Geophys. Res. 125, $653 \quad \mathrm{e} 06035(2020)$.

654 55. Yada, T. et al. Preliminary analysis of the Hayabusa2 samples returned from C-type 655 asteroid Ryugu. Nat. Astron. (2021) doi:10.1038/s41550-021-01550-6.

656 56. Villarreal, M. N. Understanding the Interiors of Vesta and Ceres Through Their 657 Interactions with the Solar Wind. (University of California Los Angeles, 2018).

658

659 